\title{
The Performance of QPSK in Low-SNR Interference Channels
}

\author{
Moritz Wiese ${ }^{\dagger}$, Frederic Knabe*, Johannes Georg Klotz* and Aydin Sezgin*‡ \\ ${ }^{\dagger}$ Heinrich-Hertz-Chair for Mobile Communications, Technical University of Berlin, \\ Einsteinufer 25, 10587 Berlin, Germany, Email: moritz.wiese@mk.tu-berlin.de \\ * Institute of Telecommunications and Applied Information Theory, Ulm University, \\ Albert-Einstein-Allee 43, 89081 Ulm, Germany, Email: \{frederic.knabe, johannes.klotz, aydin.sezgin\}@uni-ulm.de \\ $\ddagger$ Emmy-Noether Research Group on Wireless Networks
}

\begin{abstract}
We investigate the low-SNR sum rate performance of QPSK for symmetric interference channels. The QPSK performance is described by the minimum energy per bit and the wideband slope pertaining to the sum capacity. Comparing this with the minimum energy per bit and wideband slope of corresponding interference channels using optimal inputs, we find that QPSK achieves optimal performance in all of the cases where exact sum capacities are known. We also show that a simplified Han-Kobayashi scheme is suboptimal in the low-SNR regime when the input alphabet is the whole set of complex numbers.
\end{abstract}

\section{INTRODUCTION}

In modern cellular systems, interference is the main factor which limits performance. Information theory models this situation using the interference channel (IC), in which several transmitter-receiver pairs communicate and mutually disturb each other's receptions. Hence, understanding this channel is relevant for practical issues. However, the exact capacity region of this channel is still unknown. Recently, due to increased effort from the research community, considerable progress has been made in understanding the IC. In [1], the capacity of the two-user IC was characterized up to one bit by using different schemes depending on the channel parameters.

In certain regimes, the sum capacity can be determined exactly for the symmetric $K$-user IC and two simpler modifications, the symmetric one-to-many (OTM) IC and the symmetric many-to-one (MTO) IC. These regimes are: 1) The regime where interference does not harm the capacity and each user achieves single-user performance. This is called the "very strong interference regime". A condition when the symmetric IC using optimal inputs is in this regime is given in [2]. 2) The "noisy interference regime", defined as the regime where treating interference as noise is optimal. For the symmetric two-user, OTM, and MTO-ICs, it was determined in [3] when they are in the noisy interference regime. Further, exact sum capacity expressions were found in [3] for these channels in the noisy interference regime.

In this paper, we are interested in the low-SNR sum rate performance of symmetric ICs using QPSK inputs. It was shown in [4] that QPSK achieves optimal performance for degraded broadcast channels, prompting the question whether this is the case for symmetric ICs as well. In the lowSNR regime, the method of finding the minimum energy per bit and the wideband slope developed in [5] has proved to give relevant evidence on the performance. This method was already used in [6] to characterize the wideband performance of general two-user ICs using optimal inputs. We apply it here to treat the low-SNR sum rate performance of symmetric ICs using QPSK.

The main result of this paper is that in the low-SNR regime, QPSK inputs are optimal in those regions where the exact sum rate performance of symmetric ICs using optimal inputs is known. As described in the second paragraph, this is the case for the symmetric IC in the very strong interference regime. This is also the case for the symmetric two-user, MTO, and OTM-IC in the noisy interference regime. (It was already established in [7] that the noisy interference regimes of the symmetric MTO-IC using QPSK and of the symmetric MTOIC using optimal inputs coincide for all values of SNR.)

Except for the two-user MTO and OTM-IC, there remains a region between the very strong interference regime and the noisy interference regime in the low-SNR regime. We consider the two-user IC in this region, using the whole set of complex numbers as input alphabet. We show that a variant of simplified Han-Kobayashi transmission is suboptimal in this case. As an optimal scheme is unknown, QPSK optimality cannot be shown in this region.

The paper is organized as follows: the various channel models as well as the performance measures are defined in the next section. In Section III, the case where the input alphabet is the whole set of complex numbers is considered. We evaluate the low-SNR performance of optimal schemes and of the simplified Han-Kobayashi scheme. Section IV shows the optimality of using QPSK inputs for the low-SNR sum rate performance, where it can be compared with the optimal performance derived in the previous section. Finally, we conclude the paper in Section V.

\section{Problem Formulation}

\section{A. System Description}

In the general transmission scenario that we consider, each transmitter $k$ wants to send a message to its corresponding receiver $k$, where $k \in\{1, \ldots, K\}$. Unfortunately, the receivers not only receive the messages from their desired 


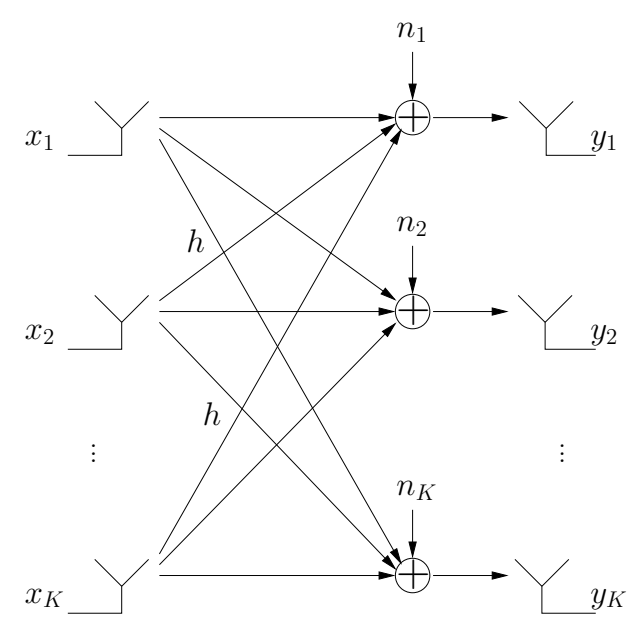

Fig. 1. The $K$-user symmetric interference channel

transmitters, but also the signals from all other transmitters $j \in\{1, \ldots, K\} \backslash k$, which act as interference. This scenario is referred to as the $K$-user interference channel (IC). In this paper, we assume this channel to be symmetric and in standard form [8], i.e., the desired signal paths are assumed to have an equal channel gain of 1 , while the channel gain of all interfering paths is $h$.

Thus, the $k$ th receiving station receives

$$
y_{k}=x_{k}+\sum_{\substack{i=1 \\ i \neq k}}^{K} h x_{i}+n_{k},
$$

where $x_{k}$ is the transmit symbol sent by user $k$, which has to fulfill the power constraint $\mathrm{E}\left(\left|x_{k}\right|^{2}\right) \leq P$ and $n_{k}$ is a Gaussian noise value. Throughout this paper we will assume that the Gaussian noise has unit variance and thus

$$
\mathrm{SNR}=P .
$$

The channel model (1) is the basis for our considerations in this paper. However, in the noisy interference regime, we will focus on three important special cases of the $K$-user IC: The two-user IC, the many-to-one (MTO) IC and the one-to-many (OTM) IC. The two-user IC is obtained from (1) by simply setting $K=2$, while in the MTO-IC and OTM-IC we have $K \geq 2$ users but not all possible interference paths present. In the MTO-IC, only one receiver (w.l.o.g. receiver 1) suffers from interference

$$
\begin{aligned}
& y_{1}=x_{1}+\sum_{i=2}^{K} h x_{i}+n_{1} \\
& y_{k}=x_{k}+n_{k} \quad k=2, \ldots, K,
\end{aligned}
$$

while in the OTM-IC only one transmitter (w.l.o.g. transmitter 1) interferes all other transmissions

$$
\begin{aligned}
& y_{1}=x_{1}+n_{1} \\
& y_{k}=x_{k}+h x_{1}+n_{k} \quad k=2, \ldots, K .
\end{aligned}
$$

In all considered channels we will use complex inputs alphabets and channel parameters, i.e. $x_{i}, y_{i}, h \in \mathbb{C}(i=1, \ldots, K)$. Since some of our references use real channel inputs and parameters, this has to be considered when comparing the expressions of the channel capacity.

\section{B. Minimum Energy per Bit and Wideband Slope}

In this paper, we will consider systems that operate at a low SNR level. For those systems the spectral efficiency $C\left(\frac{E_{b}}{N_{0}}\right)$ is a central performance measure [5]. It is defined as the ratio of the transmission rate $R$ and the bandwidth $B$ and its argument is the energy per information bit to noise ratio $\frac{E_{b}}{N_{0}}$

$$
\mathrm{C}\left(\frac{E_{b}}{N_{0}}\right)=\frac{R}{B} \text {. }
$$

The spectral efficiency is equal to the channel capacity $\mathrm{C}\left(\frac{E_{b}}{N_{0}}\right)=C(P)$, but nevertheless it makes sense to distinguish them. We use the italic $C(P)$ to identify the channel capacity as function of $P$, while we use the sans-serif $\mathrm{C}\left(\frac{E_{b}}{N_{0}}\right)$ to identify the spectral efficiency as a function of $\frac{E_{b}}{N_{0}}$. Since we consider the sum-capacity $C^{\text {sum }}(P)$ instead of single-user capacities we define the sum spectral efficiency in analogy

$$
\mathrm{C}^{\text {sum }}\left(\frac{E_{b, a v}}{N_{0}}\right)=C^{\text {sum }}(P),
$$

where $\frac{E_{b, a v}}{N_{0}}$ is now the average energy per information bit to noise ratio. Taking this average is necessary since in contrast to $P$, which is equal for all transmissions due to the assumed symmetry, the energy per information bit is not the same for all $K$ transmissions in general. The relation between $\frac{E_{b, a v}}{N_{0}}$ and $P$ is obtained by, cf. [5]

$$
\frac{E_{b, a v}}{N_{0}} \frac{C^{\mathrm{sum}}(P)}{K}=P .
$$

Since $C^{\mathrm{sum}}(P)$ is a monotonically increasing concave function, the minimum average energy per bit is achieved as $P$ approaches zero. Thus, if we want to compare different transmission strategies at low SNR level, two aspects can be considered. First, we can compare the minimum average energies per bit, that are necessary for transmission. In analogy to [5], they can be calculated as

$$
{\frac{E_{b, a v}}{N_{0}}}_{\min }=\frac{K \cdot \log _{e} 2}{\dot{C}^{\operatorname{sum}}(0)} .
$$

Second, if the minimum energies per bit are equal, the slopes of the sum spectral efficiency functions at the minimum average energies per bit can be calculated as

$$
\mathcal{S}_{0}=-2 \frac{\dot{C}^{\text {sum }}(0)^{2}}{\ddot{C}^{\text {sum }}(0)}
$$

in $b i t / s / H z /(3 \mathrm{~dB})$. These slopes can be interpreted as a measure for the sum spectral efficiency gain if the transmit power is increased from zero to a small non-zero value. For simplicity of notation, we will just use the term slope, where it is obvious from the context that we mean the slope of the sum spectral efficiency. 


\section{Optimal SCHEMES FOR THE IC AT LOW SNR}

\section{A. Very Strong Interference}

The first to observe that the full single-user rates can be achieved if interference is strong enough was Carleial [9]. We ask here how large $|h|$ needs to be in the low-SNR regime in order for every transmitter/receiver pair to achieve the full single-user performance. The regime where this is possible is called the very strong interference regime. It was noted in [2] that the symmetric $K$-user IC with optimal inputs is in the very strong interference regime if

$$
|h|^{2} \geq \frac{\left((1+P)^{K-1}-1\right)(1+P)}{(K-1) P},
$$

cf. [2], eq. (5). Clearly, for $P \rightarrow 0$, this condition reduces to

$$
|h|^{2}>\frac{K-1}{K-1}=1 .
$$

The way to achieve the single-user rates is for each receiver to decode the interfering signals first while treating the desired signal as noise. The desired signal is decoded in a second step. From [5], it is known that if the transmitter-receiver pairs achieve the single-user rates, then each of them achieves the same $\frac{E_{b}}{N_{0} \text { min }}$ of $\log _{e} 2=-1.59 \mathrm{~dB}$ and the same wideband slope of 2 as an undisturbed AWGN channel. Hence by (10), ${\frac{E_{b, a v}}{N_{0}}}_{\text {min }}$ equals $-1.59 \mathrm{~dB}$ as well. Further, the wideband slope achievable by the sum rate equals

$$
\mathcal{S}^{\text {sum }}=2 K \text {. }
$$

If the $\mathrm{K}$-user IC is in the very strong interference regime, then so are the OTM-IC and the MTO-IC. Thus, for these channels, one has the same sufficient condition $|h|^{2}>1$ for the very strong interference regime.

In [1], the "strong interference regime" is characterized as the region where $|h| \geq 1$ but the IC is not in the very strong interference regime. The very strong interference condition (13) implies that the strong interference region vanishes in the low-SNR regime.

Note that the special case of a two-user IC satisfying (13) was already treated in [6]. Further note that in [2], another bound on $|h|$ for the symmetric IC to be in the very strong interference is given. This bound is considerably tighter in the high-SNR regime. However, it tends to infinity as $P \rightarrow 0$, so it does not apply in the low-SNR regime considered here. Moreover, the way of achieving the single-user capacities is much more involved for the tightened scheme.

\section{B. Noisy interference}

Now, we consider the opposite case, where $|h|$ is small. We treat the OTM and MTO as well as the two-user symmetric IC.

1) MTO-IC: Specializing [3, Theorem 4] to the symmetric MTO-case, one obtains that for

$$
|h|^{2} \leq \frac{1}{K-1},
$$

treating interference as noise achieves the sum capacity, which is given by

$$
\begin{aligned}
& C_{\mathrm{MTO}}^{\mathrm{sum}}(P)= \\
& \log \left(1+\frac{P}{(K-1)|h|^{2} P+1}\right)+(K-1) \log (1+P) .
\end{aligned}
$$

Using (10) and (11), it follows that the minimum average energy per bit and the wideband slope equal

$$
{\frac{E_{b, a v}}{N_{0}}}_{\text {min }}=\log _{e} 2, \quad \mathcal{S}_{\mathrm{MTO}}^{\text {sum }}=\frac{2 K}{1+2|h|^{2}-\frac{2|h|^{2}}{K}} .
$$

2) OTM-IC: Here again, we only treat the symmetric case. [3, Theorem 5] says that for

$$
(K-1) \frac{|h|^{2}(P+1)}{|h|^{2} P+1} \leq 1
$$

the sum capacity $C_{\mathrm{OTM}}^{\mathrm{sum}}$ of the symmetric OTM-IC can be achieved by treating interference as noise. The sum capacity equals

$$
C_{\mathrm{OTM}}^{\mathrm{sum}}(P)=\log (1+P)+(K-1) \log \left(1+\frac{P}{|h|^{2} P+1}\right) .
$$

As $P \rightarrow 0$, condition (18) transforms into

$$
|h|^{2} \leq \frac{1}{K-1},
$$

and it should be noted that this is the same condition as (15) for the MTO-IC case. Using (19), one sees immediately that if (18) holds, then the minimum average energy per bit and the wideband slope are the same as in (17).

3) The two-user IC: [3, Theorem 1] states that if $h$ satisfies the condition

$$
|h|\left(1+|h|^{2} P\right) \leq \frac{1}{2},
$$

then treating interference as noise is optimal for achieving the sum rate

$$
C_{2 \mathrm{IC}}^{\text {sum }}(P)=2 \log \left(1+\frac{P}{1+|h|^{2} P}\right) .
$$

In the low-SNR regime, condition (21) becomes $|h| \leq 1 / 2$, or

$$
|h|^{2} \leq \frac{1}{4}
$$

Proceeding as in the previous subsection, one obtains for the average minimum energy per bit and the wideband slope $\mathcal{S}_{2 \mathrm{IC}}^{\text {sum }}$ that

$$
{\frac{E_{b, a v}}{N_{0} \min }}=\log _{e} 2 ; \quad \mathcal{S}_{2 \mathrm{IC}}^{\text {sum }}=\frac{4}{1+2|h|^{2}} .
$$

The minimum average energy per bit is the same as the minimum average energy per bit for the two user MTOIC. (Clearly, the two-user MTO-IC is equivalent to the twouser OTM-IC. This channel is also called the Z-IC.) But note that the region determined by (21) is smaller than the corresponding region for the $Z$-IC determined in (15) (and 
(20)). For $|h|^{2} \leq 1 / 4$, one sees that the wideband slope of the two-user IC differs from the slope of the $Z$-IC by a factor of

$$
\frac{\mathcal{S}_{2 \mathrm{IC}}^{\text {sum }}}{\mathcal{S}_{\text {MTO }}^{\text {sum }}}=\frac{1+|h|^{2}}{1+2|h|^{2}} \in\left[\frac{5}{6}, 1\right] \text {. }
$$

The maximum is attained for the trivial case of no interference, in which case the channels are equivalent. Still, one observes that in the regime determined by (23), the performances are surprisingly close.

\section{Digression: The two-user IC in the intermediate regime}

It was shown in Subsection III-A that the two-user IC is in the very strong interference regime for $P \rightarrow 0$ if $|h| \geq 1$. In Subsection III-B it was observed that for small $|h|$, it is in the noisy interference regime. For the intermediate region, to our knowledge, optimal schemes are unknown (recall that the strong interference region vanishes as $P \rightarrow 0$ ). One does not know whether there is any strategy which achieves the same average energy per bit as and a larger wideband slope than TDMA if $1 / 2 \leq|h|^{2} \leq 1$. (For $|h|^{2} \leq 1 / 2$, it was shown in [6] that treating interference as noise is better, and as we have seen, this is even optimal for $|h|^{2} \leq 1 / 4$.)

One could be tempted to try a simplified Han-Kobayashi scheme. (The name of the scheme goes back to [10].) In the version we consider here, both users have the same number of messages. Both of them partition their message set into two sets in the same way. The first set thus obtained is the set of private messages, consisting of messages decodable only by the intended receiver. The other set (of common messages) consists of messages decodable by both receivers. The encoding/decoding process is as follows: the set of private messages is assigned power $\alpha P, 0 \leq \alpha \leq 1$, so the set of common messages gets $(1-\alpha) P$. (This is the main difference to the simplified Han-Kobayashi scheme used in [1], where the common message is transmitted at power $1 /|h|^{2}$. As this requires a minimum positive amount of power to be spent, such a scheme cannot be applied in the low-SNR regime.) In this way, every transmitter is split up into two virtual transmitters, one for the common and one for the private part. First, one decodes the common messages, treating the private messages as noise. Then each receiver cancels the interference caused by the common messages and decodes the private message intended for him, treating the other private message as noise. Thus the sum rate of the common messages must satisfy the constraints

$$
R_{c, 1}+R_{c, 2} \leq \log \left(1+\frac{(1-\alpha)\left(1+|h|^{2}\right) P}{1+\alpha\left(1+|h|^{2}\right) P}\right),
$$

which is the sum rate constraint for each of the MACs formed by the common messages and each of the receivers, and

$$
R_{c, 1}+R_{c, 2} \leq 2 \log \left(1+\frac{(1-\alpha)|h|^{2} P}{1+\alpha\left(1+|h|^{2}\right) P}\right),
$$

which is the sum of the single-user constraints. The private rate constraint is

$$
R_{p} \leq \log \left(1+\frac{\alpha P}{1+\alpha|h|^{2} P}\right),
$$

having canceled the interference from the common messages and treating the other private message as noise. Thus a sum rate

$$
\begin{aligned}
& R_{H K}(P) \leq 2 \log \left(1+\frac{\alpha P}{1+\alpha|h|^{2} P}\right) \\
&+\min \left\{\log \left(1+\frac{(1-\alpha)\left(1+|h|^{2}\right) P}{1+\alpha\left(1+|h|^{2}\right) P}\right),\right. \\
&\left.2 \log \left(1+\frac{(1-\alpha)|h|^{2} P}{1+\alpha\left(1+|h|^{2}\right) P}\right)\right\}
\end{aligned}
$$

can be achieved. One sees immediately that for small $P$, the minimum in (29) is attained by the second term. Its derivative at $P=0$ equals $2(1-\alpha)|h|^{2}$, so the derivative of $R_{H K}(P)$ at $P=0$ equals

$$
2\left(\alpha+(1-\alpha)|h|^{2}\right)
$$

For the minimum average energy per bit achieved by this simplified Han-Kobayashi scheme, this means

$$
{\frac{E_{b, a v}}{N_{0}}}_{\text {min }}=\frac{\log _{e} 2}{\alpha+(1-\alpha)|h|^{2}} \geq \log _{e} 2
$$

for $|h|^{2}<1$. Comparing this with the results in [6], which show that for every $|h|<1$ the minimum energy $\log _{e} 2$ is achievable, one sees that simplified Han-Kobayashi is wideband optimal only for $\alpha=1$. However, in this case, there is no partitioning of the messages, so in a strict sense, this is no Han-Kobayashi scheme any more.

\section{QPSK FOR THE IC AT LOW SNR}

Since it has already been shown that QPSK is optimal in low-SNR point to point and broadcast channels [4,5], it is natural to consider QPSK also for transmissions over interference channels at low SNR. Therefore, we will derive a general Taylor-series of the QPSK mutual information in section IV-A. This expression will be used to calculate the slope of the sum rate in the noisy (section IV-B) and very strong interference regime (section IV-C).

\section{A. Taylor-series of Mutual Information}

To obtain the wideband slope and average minimum energy per bit of a QPSK transmission, we have to consider the mutual information of the channel input and output instead of the channel capacity. Although the mutual information terms of a Gaussian IC with QPSK inputs are complicated and hard to handle, it is possible to compute a Taylor-series of a lower bound on the mutual information at $P \rightarrow 0$. With this Taylorseries and (10), (11) the computation of average minimum energy per bit and slope is simple.

Let $X, X_{I}, N$ and $Y$ be complex random variables denoting the channel input, the total interference at the receiver, the channel noise and the channel output, such that

$$
Y=a X+X_{I}+N
$$

where $a \in \mathbb{C}, \mathrm{E}\left(\left|X^{2}\right|\right)=P, \mathrm{E}\left(\left|X_{I}\right|^{2}\right)=P S, S \in \mathbb{R}$ and $N \sim \mathcal{C N}(0,1)$. Moreover let $Y_{G}$ and $X_{I, G} \sim \mathcal{C N}(0, P S)$ be random variables that fulfill

$$
Y_{G}=a X+X_{I, G}+N .
$$


If we treat interference as noise, it is known from [11] that if the SNR is low, we have

$$
I_{4, G}(P):=I\left(X ; Y_{G}\right) \leq I(X ; Y)=: I_{4}(P),
$$

i.e., the mutual information of $Y$ and $X$ is lower bounded by the case where the interference is Gaussian and has the same power. In order to write the mutual information terms as a function of $P$, we use the term $I_{4, G}(P)$ for the left side and $I_{4}(P)$ for the right side of (34), where the index 4 indicates that we use QPSK as transmission scheme. Moreover, with

$$
I(X ; Y)=E_{x}\left\{D\left(P_{Y \mid X=x} \| P_{Y \mid X=0}\right)\right\}-D\left(P_{Y} \| P_{Y \mid X=0}\right),
$$

we can express the mutual information as the combination of two Kullback-Leibler distances. This has already been used for expanding the mutual information to a Taylor-series $[4,5]$.

Since (33) describes a simple AWGN channel with QPSK inputs and noise variance $1+P S$, its mutual information is equal to that achieved by two independent BPSK channels with inputs $\pm \sqrt{\frac{P}{2}}$ and the same noise variance. The SNR of this BPSK channel is half that of the original channel and thus

$$
I_{4, G}(P)=2 I_{2, G}(P / 2),
$$

where $I_{2, G}(P)$ denotes the mutual information of one BPSK channel. Using (35) and proceeding similarly as [4,5], we can write the mutual information as

$$
I_{2, G}(P)=|a|^{2} P-2 S|a|^{2} P^{2}-|a|^{4} P^{2}+o\left(P^{2}\right) .
$$

To summarize the above equations, we have seen that the mutual information of $X$ and $Y$ in the channel model (32) can be lower bounded by the Taylor-series

$$
I_{4}(P) \geq|a|^{2} P-S|a|^{2} P^{2}-\frac{1}{2}|a|^{4} P^{2}+o\left(P^{2}\right)
$$

at $P \rightarrow 0$ if we use QPSK as input alphabets. By setting $a=1$ we can use this to calculate an upper bound for the required minimum energy per bit of the transmission from transmitter $k$ to receiver $k$ with $k \in\{1, \ldots, K\}$ in any of the considered channels. With (10), it can be observed that the minimum required energy per bit is upper bounded by $\log _{e} 2=-1.59 \mathrm{~dB}$, independent of the interference power $P S$. Thus, we have

$$
\frac{E_{b, a v}}{N_{0}} \leq \log _{e} 2=-1.59 \mathrm{~dB}
$$

for all considered channels. Moreover, since the same value is achieved by optimal inputs (c.f. (24)) this bound has to be tight.

\section{B. QPSK in the noisy interference regime}

In this subsection, we will use (38) to obtain lower bounds on the sum mutual information and the corresponding slope expressions in the noisy interference regime of the considered ICs. This is done by setting $S$ according to the channel parameters. The tightness of these bounds is directly established since the slopes will be equal to those achieved by Gaussian alphabets, which are optimal.
1) 2-User IC: For obtaining a lower bound on the slope of the two-user interference channel we have to set $S=|h|^{2}$, $a=1$ and obtain

$$
I_{4,2 \mathrm{IC}}(P) \geq P-|h|^{2} P^{2}-\frac{1}{2} P^{2}+o\left(P^{2}\right)
$$

for the mutual information of both users. By using (11) this leads to a slope of

$$
\mathcal{S}_{4,2 \mathrm{IC}}^{\mathrm{sum}} \geq \frac{4}{1+2|h|^{2}} .
$$

Since the right side of (41) equals the maximum achievable slope in (24), it is obvious that this bound is tight. Thus, we can state that if $P$ tends to zero and the noisy interference condition $|h|^{2} \leq \frac{1}{4}$ is fulfilled, using QPSK is optimal in the 2-user IC.

2) MTO-IC: Considering the MTO-IC, we have to set $S=$ $(K-1)|h|^{2}, a=1$ for the first user and $S=0, a=1$ for all other users $k=2, \ldots, K$, i.e.,

$$
\begin{aligned}
& I_{4, \text { OTM }, 1}(P) \geq P-(K-1)|h|^{2} P^{2}-\frac{1}{2} P^{2}+o\left(P^{2}\right) \\
& I_{4, \text { OTM }, k}(P) \geq P-\frac{1}{2} P^{2}+o\left(P^{2}\right) .
\end{aligned}
$$

Summing up these mutual information terms and calculating the slope with (11) results in

$$
\mathcal{S}_{4, \mathrm{MTO}}^{\text {sum }} \geq \frac{2 K}{1+2|h|^{2}-\frac{2|h|^{2}}{K}} .
$$

Comparing this lower bound to (17) directly proves that the bound is tight in the noisy interference regime. Thus, it is shown that QPSK and treating interference as noise achieves optimality in the low-SNR regime of MTO-ICs if $|h|^{2} \leq(K-1)^{-1}$. This threshold is consistent with the observations in [7] for non-zero SNR.

3) OTM-IC: In contrast to the MTO-IC, we have only one interference free user in the OTM-IC. All other users are disturbed by the transmission of the first transmitter, such that $S=0, a=1$ for user one and $S=|h|^{2}, a=1$ for all other users $k=2, \ldots, K$. Thus, we obtain for the Taylor-series of the mutual information

$$
\begin{aligned}
& I_{4, \mathrm{MTO}, 1}(P) \geq P-\frac{1}{2} P^{2}+o\left(P^{2}\right) \\
& I_{4, \mathrm{MTO}, k}(P) \geq P-\left(|h|^{2}+\frac{1}{2}\right) P^{2}+o\left(P^{2}\right),
\end{aligned}
$$

which by summing those terms up and using (11) again leads to a slope of

$$
\mathcal{S}_{4, \mathrm{OTM}}^{\text {sum }} \geq \frac{2 K}{1+2|h|^{2}-\frac{2|h|^{2}}{K}} .
$$

As already observed for the Gaussian case, this is exactly the same slope as in the MTO-IC. Hence, we have shown that in this regime the MTO-IC and OTM-IC have an equal performance if QPSK is used. Moreover, the optimality of QPSK in the low-SNR and noisy interference regime of OTMICs, which is also obtained for $|h|^{2} \leq(K-1)^{-1}$, is proved. 


\section{QPSK in the very strong interference regime}

As already stated, the very strong interference regime is the regime where interference does not harm the capacity. This is ensured, if all interfering signals can be decoded before the desired signal. If this is the case, the channel of all transmitterreceiver pairs has the same performance as a single-user AWGN channel. As already shown in [5], for single-user AWGN channels QPSK achieves an optimal performance for $P \rightarrow 0$.

From (13) we know that if optimal inputs are used and $|h|>1$, the $K$-user IC is in the very strong interference regime as $P \rightarrow 0$. However, the question is if the same constraint holds if QPSK is used. We will show that this is the case and briefly describe how the interfering signals can be decoded successively. As underlying channel model we use the fully connected and symmetric $K$-user IC (1), i.e., the considered special cases (MTO-IC, OTM-IC, $K=2$ ) are already included.

If the $K$-user IC is in the very strong interference regime, each user $k$ can communicate at rate

$$
R \leq I\left(Y_{k} ; X_{k} \mid X_{1}, \ldots, X_{k-1}, X_{k+1}, \ldots, X_{k}\right),
$$

where due to symmetry the term on the right side of (48) is equal for all $k \in\{1, \ldots, K\}$. Now, it has to be ensured, that fulfilling (48) is enough to make the interfering signals decodable at each receiver. Without loss of generality we consider the decoding process at receiver $K$. The decoding order shall be such that in step $i \in\{1, \ldots, K-1\}$, we decode $X_{i}$ while treating the signals $X_{i+1}, \ldots, X_{K}$ as noise. Thus, for ensuring successful decoding of $X_{i}$ at receiver $\mathrm{K}$, the condition

$$
R \leq I\left(Y_{K} ; X_{i} \mid X_{1}, \ldots, X_{i-1}\right)
$$

has to be fulfilled. And for ensuring the channel to be in the very strong interference regime (49) has to be an implication of (48). This is the case if for $i \in\{1, \ldots, K-1\}$

$$
I\left(Y_{K} ; X_{i} \mid X_{1}, \ldots, X_{i-1}\right) \geq I\left(Y_{K} ; X_{K} \mid X_{1}, \ldots, X_{K-1}\right) .
$$

As $P \rightarrow 0$, it is sufficient to consider the first term of the Taylor series at $P=0$ on both sides of (50). For the right side this term is known to be $P+o(P)$ [5]. For the left side we can use the channel model (32) with $a=h$ and $S=1+h(K-i)$. With (38) we obtain

$$
I\left(Y_{K} ; X_{i} \mid X_{1}, \ldots, X_{i-1}\right) \geq|h|^{2} P+o(P)
$$

as lower bound. Thus, comparing the Taylor-series of both sides of (50), we obtain the condition

$$
|h| \geq 1
$$

for the $K$-user IC to be in the very strong interference regime, which is the same as for Gaussian alphabets.

\section{CONCLUSiON}

In this paper, we investigated the $K$-user symmetric IC at low SNR. Therefore, we used the minimum average energy per bit and the wideband slope defined in [5] as performance measure. We could show that in the very strong interference regime, we can use QPSK inputs and still achieve optimal performance. In the noisy interference regime, we considered three special cases of the $K$-user symmetric IC: The 2-user IC, the OTM-IC and the MTO-IC. It could be shown, that QPSK input alphabets can be used without loss of performance in these channels as the power tends to zero. Moreover, we have seen that near zero SNR, the MTO-IC and OTM-IC have an equal performance in the noisy interference regime. For the two-user IC, we also considered the regime between the noisy and the very strong interference regime. It was known from [1], that the message splitting scheme of Han and Kobayashi [10] achieves sum rates within one bit of the capacity in this regime. However, we have shown that this kind of message splitting is suboptimal at low SNR.

\section{ACKNOWLEDGMENT}

This work was supported by the German research council "Deutsche Forschungsgemeinschaft" (DFG) under grants Bo 1734/15-1, Bo 867/18-1, Bo 867/19-1 and Se 1697/3-1.

\section{REFERENCES}

[1] R. Etkin, D. Tse, and H. Wang, "Gaussian Interference Channel Capacity to Within One Bit," Information Theory, IEEE Transactions on, vol. 54, no. 12, pp. 5534-5562, 2008. [Online]. Available: http://dx.doi.org/10.1109/TIT.2008.2006447

[2] S. Sridharan, A. Jafarian, S. Vishwanath, and S. Jafar, "Capacity of Symmetric K-User Gaussian Very Strong Interference Channels," in IEEE GLOBECOM, New Orleans, LA, USA, 2008.

[3] V. S. Annapureddy and V. V. Veeravalli, "Gaussian Interference Networks: Sum Capacity in the Low-Interference Regime and New Outer Bounds on the Capacity Region," Information Theory, IEEE Transactions on, vol. 55, no. 7, pp. 3032-3050, June 2009. [Online]. Available: http://dx.doi.org/10.1109/TIT.2009.2021380

[4] F. Knabe, M. Wiese, C. Huppert, and J. Klotz, "The Wideband Slope Region of BPSK and QPSK for Broadcast Channels in the Low-Power Regime," in 2009 Information Theory Workshop, Taormina, Taormina, Sicily, Italy, 2009.

[5] S. Verdu, "Spectral Efficiency in the Wideband Regime," Information Theory, IEEE Transactions on, vol. 48, no. 6, pp. 1319-1343, 2002. [Online]. Available: http://dx.doi.org/10.1109/TIT.2002.1003824

[6] G. Caire, D. Tuninetti, and S. Verdu, "Suboptimality of TDMA in the Low-Power Regime," Information Theory, IEEE Transactions on, vol. 50, no. 4, pp. 608-620, 2004. [Online]. Available: http://dx.doi.org/10.1109/TIT.2004.825003

[7] V. R. Cadambe and S. A. Jafar, "Interference Alignment and a Noisy Interference Regime for Many-to-One Interference Channels," e-print arXiv:0912.3029.

[8] A. Carleial, "Interference Channels," Information Theory, IEEE Transactions on, vol. 24, no. 1, pp. 60-70, January 2003. [Online]. Available: http://ieeexplore.iee.org/xpls/abs_all.jsp?arnumber $=1055812$

[9] — - "A Case Where Interference Does Not Reduce Capacity," Information Theory, IEEE Transactions on, vol. 21, no. 5, pp. 569-570, 1975.

[10] T. Han and K. Kobayashi, "A New Achievable Rate Region for the Interference Channel," Information Theory, IEEE Transactions on, vol. IT-27, no. 1, pp. 49-60, 1981.

[11] C. Cahn, "Worst Interference for Coherent Binary Channel (Corresp.)," Information Theory, IEEE Transactions on, vol. 17, no. 2, March 1971. 